\title{
Pattern distributions in Dyck paths with a first return decomposition constrained by height
}

\author{
Jean-Luc Baril ${ }^{*}$ Richard Genestier $\dagger^{\dagger}$ and Sergey Kirgizov ${ }^{\ddagger}$ \\ LIB, Univ. Bourgogne Franche-Comté, France
}

May 19, 2020

\begin{abstract}
We provide generating functions for the popularity and the distribution of patterns of length at most three over the set of Dyck paths having a first return decomposition constrained by height.
\end{abstract}

Keywords: Dyck and Motzkin paths, pattern statistic, distribution, popularity

\section{Introduction and notation}

Dyck paths with a constrained first return decomposition were introduced in [4] where the authors present both enumerative results using generating functions and a constructive bijection with the set of Motzkin paths. In [5], a similar study has been conducted for Motzkin, 2-colored Motzkin, Schröder and Riordan paths. In the literature, many papers deal with the enumeration of classical Dyck paths according to different parameters, e.g. length, number of peaks, valleys, double rises and other pattern occurrences [10, 15, 16, 18, 19, 20, 21, 24]. Restricted classes of Dyck paths have also been considered, for instance Barcucci et al. [2] consider Dyck paths having a non-decreasing height sequence of valleys (see also [8, 9]). Other papers deal with Motzkin paths using similar methods [3, 6, 11, 12, 17, 22, 25]. Motzkin and Catalan numbers appear alongside in many situations [11] and several one-to-one correspondences exist between restricted Dyck paths and Motzkin paths. For instance, Dyck paths avoiding a triple rise are enumerated by the Motzkin numbers [7].

In this paper, we focus on the distribution and the popularity of patterns of length at most three in constrained Dyck paths defined in [4]. Our method consists in showing how patterns are getting transferred from constrained Dyck paths to Motzkin paths, which settles us in a more suitable ground in order to provide generating functions for the distribution and the popularity.

A Motzkin path of length $n \geqslant 0$ is a lattice path consisting of flat steps $F=(1,0)$, up steps $U=(1,1)$ and down steps $D=(1,-1)$, starting at $(0,0)$,

\footnotetext{
${ }^{*}$ Electronic address: barj1@u-bourgogne.fr

${ }^{\dagger}$ Electronic address: Richard.Genestier@u-bourgogne.fr

${ }^{\ddagger}$ Electronic address: sergey.kirgizov@u-bourgogne.fr; Corresponding author
} 
ending at $(n, 0)$ and never going below the $x$-axis. For $n \geqslant 0$, we denote by $\mathcal{M}_{n}$ the set of all Motzkin paths of length $n$ and we set $\mathcal{M}=\bigcup_{n \geqslant 0} \mathcal{M}_{n}$. A Motzkin path of length $2 n$ with no flat steps is a Dyck path of semilength $n$. For $n \geqslant 0$, let $\mathcal{D}_{n}$ be the set of all Dyck paths of semilength $n$ and $\mathcal{D}=\bigcup_{n \geqslant 0} \mathcal{D}_{n}$. The cardinality of $\mathcal{D}_{n}$ is given by the $n$th Catalan number $c_{n}=\frac{1}{n+1}\left(\begin{array}{c}2 n \\ n\end{array}\right)$, which is the general term of the sequence A000108 in the On-line Encyclopedia of Integer Sequences of N.J.A. Sloane [23]. The cardinality of $\mathcal{M}_{n}$ is given by the $n$th Motzkin number $\sum_{k=0}^{\lfloor n / 2\rfloor}\left(\begin{array}{c}n \\ 2 k\end{array}\right) c_{k}$ (see A000108 in [23]).

Any non-empty Dyck path $P \in \mathcal{D}$ has a unique first return decomposition [10] of the form $P=U \alpha D \beta$ where $\alpha$ and $\beta$ are two Dyck paths in $\mathcal{D}$. In [4], the authors introduced the set $\mathcal{D}^{h, \geqslant}$ constituted of the empty Dyck path and the Dyck paths in $\mathcal{D}$ having a first return decomposition satisfying

$$
h(U \alpha D) \geqslant h(\beta)
$$

where $\alpha, \beta \in \mathcal{D}^{h, \geqslant}$ and $h$ returns the maximal height of a Dyck path. For $n \geqslant 0$, let $\mathcal{D}_{n}^{h, \geqslant}$ be the subset of Dyck paths of semilength $n$ in $\mathcal{D}^{h, \geqslant}$. For instance, $\mathcal{D}_{3}^{h, \geqslant}$ consists of four Dyck paths $U D U D U D, U U D D U D, U U D U D D$ and $U U U D D D$. In [4], the authors prove, using generating functions, that $\mathcal{D}_{n}^{h, \geqslant}$ and $\mathcal{M}_{n}$ have the same cardinality, and they present also the following bijection $\phi$ between these sets.

For $P \in \mathcal{D}^{h, \geqslant}$,

$$
\phi(P)= \begin{cases}\epsilon & \text { if } P=\epsilon \\ \phi(\alpha) F & \text { if } P=\alpha U D \\ \phi(\alpha) \phi(\gamma) U \phi(\beta) D & \text { if } P=\alpha U U \beta D \gamma D .\end{cases}
$$

For instance, the images by $\phi$ of $U D U D U D, U U D D U D, U U D U D D, U U U D D D$, $U U U U D D D D U U U D D U D D$ are respectively $F F F, U D F, F U D, U F D$ and $U U D D F U F D$. We refer to Figure 1 for an illustration of this mapping.

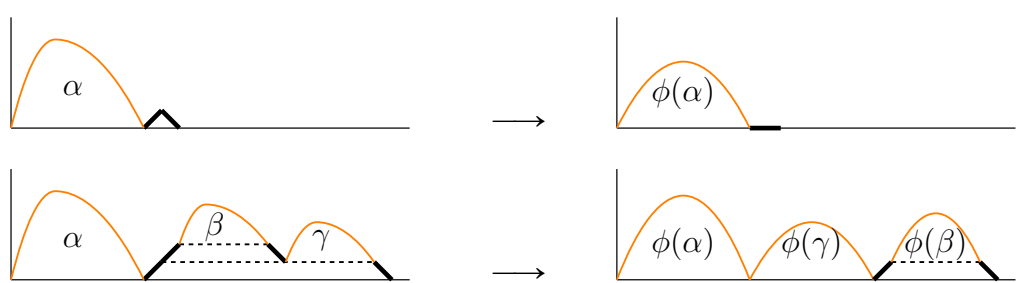

Figure 1: Illustration of the bijection $\phi$ between $\mathcal{D}_{n}^{h, \geqslant}$ and $\mathcal{M}_{n}$.

A statistic on a set $S$ of paths is an association of an integer in $\mathbb{Z}$ to each path in $S$. For instance the map that returns the number of steps is a statistic, and we denote by 1 (resp. 0) the constant statistic that sends any path to 1 (resp. 0). Let $\mathcal{S}$ be the set of all statistics on a set $S$. For $\mathrm{X}, \mathrm{Y} \in \mathcal{S}$, we define the statistic $\mathrm{X}+\mathrm{Y}$ so that $(\mathrm{X}+\mathrm{Y})(P)=\mathrm{X}(P)+\mathrm{Y}(P)$ for any $P \in S$, which endows $\mathcal{S}$ with a $\mathbb{Z}$-module structure. Let $S$ and $T$ be two sets of paths, and let $\mathcal{S}$ and $\mathcal{T}$ be the associated statistic sets. Two statistics $\mathrm{X} \in \mathcal{S}$ and $\mathrm{Y} \in \mathcal{T}$ have the same distribution if and only if there exists a bijection $f$ from $S$ to $T$ 
such that for any $P \in S$ we have $\mathrm{X}(P)=\mathrm{Y}(f(P))$. In this case, we say that $f$ transports the statistic $\mathrm{X}$ into $\mathrm{Y}$, which can be shortly written with the statistic equation $f(\mathrm{X})=\mathrm{Y}$ (or $\mathrm{X}=\mathrm{Y}$ whenever $f$ is the identity).

A pattern $X$ of length $k \geqslant 1$ occurs in a path $P$ if and only if $P$ contains $X$ as a sequence of consecutive steps. Note that other variants of pattern definition exist in the literature (see for instance [1]). From a given pattern $X$ and a set $S$ of paths, we associate the statistic $\mathrm{X}$ from $S$ to $\mathbb{N}$ such that $\mathrm{X}(P)$ is the number of occurrences of the pattern $X$ in $P$. The popularity of a pattern $X$ in $S$ is the total number of occurrences of the pattern $X$ in all paths $P \in S$, that is $\sum_{P \in S} \mathrm{X}(P)$.

For instance, if $P=U U U D D U D D$ then we have $\operatorname{UD}(P)=\mathrm{UU}(P)=2$, $\operatorname{DDD}(P)=0$. Moreover, if $S=\{U U D D, U D U D\}$ then the popularity of the pattern $U D$ in $S$ is 3. Also, for any subset $S$ of $\mathcal{M}$, we have the statistic equation $\mathrm{U}=\mathrm{D}$ since for any path in $S$ the number of up steps equals to the number of down steps. If we restrict $S$ to $\mathcal{D}_{n}\left(\right.$ resp. $\mathcal{M}_{n}$ ), then we have $\mathrm{U}+\mathrm{D}=2 \mathrm{n}$ (resp. $\mathrm{U}+\mathrm{F}+\mathrm{D}=\mathrm{n}$ ), where $\mathrm{n}$ is the constant statistic $P \mapsto n$.

Considering the above bijection $\phi$ from $\mathcal{D}_{n}^{h, \geqslant}$ to $\mathcal{M}_{n}$, the length of $\phi(P)$ is the semilength of $P$ and we easily deduce the statistic equation:

$$
\phi(\mathrm{U})=\phi(\mathrm{D})=\mathrm{U}+\mathrm{D}+\mathrm{F}=\mathrm{n} .
$$

In this paper, we present statistic equations showing how $\phi: \mathcal{D}_{n}^{h, \geqslant} \rightarrow \mathcal{M}_{n}$ transports statistics associated to patterns of length at most three into linear combinations of other statistics. Then, this allows us to conduct our enumerative study in the more natural and simpler context of Motzkin paths, while a direct study on $\mathcal{D}_{n}^{h, \geqslant}$ is complicated due to the lack of an adequate recursive decomposition. So, we use statistic equations in order to derive bivariate generating functions for the distribution of patterns of length at most three, and we deduce the pattern popularity thereafter. Section 2 deals with patterns of length 2 while Section 3 deals with patterns of length 3. Many of the resulting sequences correspond to existing entries in the On-line Encyclopedia of Integer Sequences of N.J.A. Sloane [23] enumerating a quantity of already known combinatorial structures, and we also obtain new sequences not yet known in [23].

\section{Patterns of length 2}

In this part, we provide statistic equations showing how patterns of length two behave through $\phi: \mathcal{D}_{n}^{h, \geqslant} \rightarrow \mathcal{M}_{n}$, which allows us to deduce generating functions for the distribution and the popularity of such patterns. See Table 1 for an illustration of the distributions and Table 2 for the first terms of popularity sequences.

Theorem 1. For $n \geqslant 0$, the bijection $\phi$ from $\mathcal{D}_{n}^{h, \geqslant}$ to $\mathcal{M}_{n}$ transports statistics associated to patterns of length two as follows:

$$
\begin{aligned}
\phi(\mathrm{UD}) & =\mathrm{F}+\mathrm{UD}, \\
\phi(\mathrm{UU})=\phi(\mathrm{DD}) & =\mathrm{U}+\mathrm{UU}+\mathrm{UF}, \\
\phi(\mathrm{DU}) & =\mathrm{FF}+\mathrm{FU}+\mathrm{DF}+\mathrm{DU} .
\end{aligned}
$$


Proof. For equation (2), we refer to [4].

For equation (3), we observe that for any Dyck path of semilength $n$ an up step $U$ is always followed by $U$ or $D$, which implies the equality $\mathrm{UU}+\mathrm{UD}=\mathrm{n}$ on $\mathcal{D}_{n}^{h, \geqslant}$. With a similar argument and $\mathrm{U}=\mathrm{D}$, we have $\mathrm{F}+\mathrm{U}+\mathrm{UD}+\mathrm{UU}+\mathrm{UF}=\mathrm{n}$ on $\mathcal{M}_{n}$. Using eq. (2) we obtain $\phi(\mathrm{UU})=\mathrm{n}-\phi(\mathrm{UD})=\mathrm{n}-(\mathrm{F}+\mathrm{UD})=$ $\mathrm{U}+\mathrm{UU}+\mathrm{UF}$.

For equation (4), we observe that the statistic $\mathrm{UU}+\mathrm{UD}+\mathrm{DU}+\mathrm{DD}$ equals $2 \mathrm{n}-1$ on $\mathcal{D}_{n}^{h, \geqslant}$. Using the straightforward equality $\mathrm{UU}=\mathrm{DD}$ on $\mathcal{D}_{n}$, we obtain $\mathrm{DU}=2 \mathrm{n}-1-2 \mathrm{UU}-\mathrm{UD}$. Applying the bijection $\phi$ and using eqs. (2) and (3) we obtain $\phi(\mathrm{DU})=2 \mathrm{n}-1-2(\mathrm{U}+\mathrm{UU}+\mathrm{UF})-(\mathrm{F}+\mathrm{UD})$. On the other hand, on the set of Motzkin paths of length $n$ we have the statistic equations $\mathrm{U}+\mathrm{F}+\mathrm{D}=\mathrm{n}$ and $\mathrm{FD}+\mathrm{FU}+\mathrm{FF}+\mathrm{DD}+\mathrm{DU}+\mathrm{DF}+\mathrm{UD}+\mathrm{UU}+\mathrm{UF}=\mathrm{n}-1$, which induces $2 \mathrm{n}-1=\mathrm{U}+\mathrm{F}+\mathrm{D}+\mathrm{FD}+\mathrm{FU}+\mathrm{FF}+\mathrm{DD}+\mathrm{DU}+\mathrm{DF}+\mathrm{UD}+\mathrm{UU}+\mathrm{UF}$. Also, for any Motzkin path we have $\mathrm{U}=\mathrm{D}$ which implies $\mathrm{UU}+\mathrm{UF}+\mathrm{UD}=\mathrm{UD}+\mathrm{FD}+\mathrm{DD}$, and thus $\mathrm{UU}+\mathrm{UF}=\mathrm{FD}+\mathrm{DD}$. So, combining all these equations, we obtain $\phi(\mathrm{DU})=\mathrm{U}+\mathrm{F}+\mathrm{D}+\mathrm{FD}+\mathrm{FU}+\mathrm{FF}+\mathrm{DD}+\mathrm{DU}+\mathrm{DF}+\mathrm{UD}+\mathrm{UU}+\mathrm{UF}-\mathrm{U}-$ $\mathrm{D}-\mathrm{UU}-\mathrm{UF}-\mathrm{FD}-\mathrm{DD}-\mathrm{F}-\mathrm{UD}=\mathrm{FF}+\mathrm{FU}+\mathrm{DF}+\mathrm{DU}$.

Theorem 2. The bivariate generating functions $F_{p}(x, y)$ where the coefficient of $x^{n} y^{k}$ is the number of Dyck paths in $\mathcal{D}_{n}^{h} \geqslant$ containing exactly $k$ occurrences of the pattern $p \in\{U D, U U, D D, D U\}$ are

$$
\begin{gathered}
F_{U D}(x, y)=\frac{x^{2}-x^{2} y-x y+1-\sqrt{-4 x^{2}+\left(x^{2}(y-1)+x y-1\right)^{2}}}{2 x^{2}}, \\
F_{U U}(x, y)=F_{D D}(x, y)=\frac{x^{2} y^{2}-x^{2} y-x+1-\sqrt{-4 x^{2} y^{2}+\left(x^{2} y(y-1)-x+1\right)^{2}}}{2 x^{2} y^{2}}, \\
F_{D U}(x, y)=\frac{x^{2} y-x^{2}-x y+1-\sqrt{-4 x^{2}+\left(x^{2}(y-1)+x y-1\right)^{2}}}{2 x^{2} y} .
\end{gathered}
$$

Proof. For $p=U D$, Corollary 3 in [4] provides directly the bivariate generating function as a solution of a functional equation on Motzkin paths with respect to the number of occurrences of patterns $F$ and $U D$ (see eq. (2)).

For $p=U U$, there are two ways to obtain the generating function. First, we know that $\mathrm{UU}+\mathrm{UD}=\mathrm{n}$ for Dyck paths of semilength $n$. So, we can obtain directly the generating function by calculating $F_{U D}\left(x y, \frac{1}{y}\right)$. Moreover we know that $\phi(\mathrm{UU})=\mathrm{U}+\mathrm{UU}+\mathrm{UF}$ (see eq. (3)). So, we decompose the set $\mathcal{M}$ of all Motzkin paths in the following way:

$$
\mathcal{M}=\epsilon+F \mathcal{M}+U D \mathcal{M}+U(\mathcal{M} \backslash \epsilon) D \mathcal{M} .
$$

The generating function for $\epsilon+F \mathcal{M}$ is given by $1+x M(x, y)$; the g.f. for $U D \mathcal{M}$ is $x^{2} y M(x, y)$ since $U D$ contains one occurrence of $U$; the g.f. for $U(\mathcal{M} \backslash \epsilon) D \mathcal{M}$ is $x^{2} y^{2}(M(x, y)-1) M(x, y)$ since a path in $U(\mathcal{M} \backslash \epsilon) D$ starts with $U$ and its first two steps are either $U U$ or $U F$. This induces the functional equation

$$
M(x, y)=1+x M(x, y)+x^{2} y M(x, y)+x^{2} y^{2}(M(x, y)-1) M(x, y),
$$


which also gives the expected result.

For $p=D U$, the result is obtained by using the equality $\mathrm{DU}=\mathrm{UD}-1$ and evaluating $F_{D U}(x, y)=1+\frac{F_{U D}(x, y)-F_{U D}(x, 0)}{y}$. Note that we can obtain this result using eq. (4).

Table 1 gives the number of paths in $\mathcal{D}_{n}^{h, \geqslant}$ having $k$ peaks $U D$ for $1 \leqslant n \leqslant 11$ and $1 \leqslant k \leqslant 8$. Applying standard techniques from generating function theory, we verify that the second row corresponds to the sequence of quarter-squares numbers, $\left\lfloor\left(n^{2} / 4\right)\right\rfloor$, which is the third row of Lozanić's triangle (see A034851 and [14]). The third row is a shift of the sequence A005994 corresponding to alkane numbers $l(7, n)$ from fifth row of Lozanić's triangle, which enumerates certain symmetries exhibited by chemical entities (alkane) consisting of hydrogen and carbon atoms arranged in a tree-like structure. Third diagonal corresponds to the squares, while fourth diagonal generates octahedral numbers, $n\left(2 n^{2}+1\right) / 3$ (see A005900).

\begin{tabular}{c|ccccccccccc}
$k \backslash n$ & 1 & 2 & 3 & 4 & 5 & 6 & 7 & 8 & 9 & 10 & 11 \\
\hline 1 & 1 & 1 & 1 & 1 & 1 & 1 & 1 & 1 & 1 & 1 & 1 \\
2 & & 1 & 2 & 4 & 6 & 9 & 12 & 16 & 20 & 25 & 30 \\
3 & & & 1 & 3 & 9 & 19 & 38 & 66 & 110 & 170 & 255 \\
4 & & & & 1 & 4 & 16 & 44 & 111 & 240 & 485 & 900 \\
5 & & & & & 1 & 5 & 25 & 85 & 260 & 676 & 1615 \\
6 & & & & & & 1 & 6 & 36 & 146 & 526 & 1602 \\
7 & & & & & & & 1 & 7 & 49 & 231 & 959 \\
8 & & & & & & & & 1 & 8 & 64 & 344 \\
9 & & & & & & & & & 1 & $\ldots$ & $\ldots$ \\
\hline$\Sigma$ & 1 & 2 & 4 & 9 & 21 & 51 & 127 & 323 & 835 & 2188 & 5798
\end{tabular}

Table 1: Number of paths in $\mathcal{D}_{n}^{h, \geqslant}$ having $k$ peaks $U D$, or equivalently $k-1$ valleys $D U$, or equivalently $n-k$ double rises $U U$.

Corollary 1. For $n \geqslant 0$, the popularity of pattern $p \in\{U U, U D, D D, D U\}$ in $\mathcal{D}_{n}^{h, \geqslant}$ is given by the generating function $G_{p}(x)$ :

$$
\begin{gathered}
G_{U D}(x)=\frac{(x-1) \sqrt{-3 x^{2}-2 x+1}-3 x^{2}-2 x+1}{2 x(3 x-1)}, \\
G_{U U}(x)=G_{D D}(x)=\frac{\sqrt{-3 x^{2}-2 x+1}\left(x^{2}+2 x-2\right)+x^{3}-3 x^{2}-4 x+2}{2 x^{2} \sqrt{-3 x^{2}-2 x+1}}, \\
G_{D U}(x)=\frac{\left(x^{2}-1\right) \sqrt{-3 x^{2}-2 x+1}-x^{3}-3 x^{2}-x+1}{2 x^{2} \sqrt{-3 x^{2}-2 x+1}} .
\end{gathered}
$$

Proof. The generating function $G_{p}(x)$ of popularity is directly deduced from the bivariate generating function of pattern distribution

$$
G_{p}(x)=\left.\frac{\partial F_{p}(x, y)}{\partial y}\right|_{y=1} .
$$


See Table 2 for the first terms of the popularity sequences. The popularity of the pattern $U D$ generates a shift of the sequence A025566 in [23]. As suggested in [23], the same sequence enumerates the first differences of the directed animals sequence A005773, and also Motzkin paths of length $2 n$ whose last weak valley occurs immediately after step $n$.

The popularity sequence for $D U$ is the sequence A025567. As mentioned in [13] by Ferrari and Munarini, this sequence corresponds to the number of edges in Hasse diagram of Motzkin paths, where the partial order is defined by the coverings $F F \mapsto U D, F U \mapsto U F, D F \mapsto F D, D U \mapsto F F$. This is an immediate consequence of the fact that $\phi$ maps pattern $D U$ from constrained Dyck paths to the patterns $F F, F U, D F, D U$ in Motzkin paths.

The popularity sequence for $U U$ (or $D D$ ) does not yet appear in [23].

\begin{tabular}{c|c|c} 
Pattern & Popularity sequence & OEIS \\
\hline UD & $1,3,8,22,61,171,483,1373,3923,11257,32418,93644$ & A025566 \\
DU & $0,1,4,13,40,120,356,1050,3088,9069,26620,78133$ & A025567 \\
UU, DD & $0,1,4,14,44,135,406,1211,3592,10623,31260,92488$ &
\end{tabular}

Table 2: Popularity of length two patterns in $\mathcal{D}_{n}^{h, \geqslant}$ for $1 \leqslant n \leqslant 12$.

\section{Patterns of length 3}

In this part we investigate how $\phi$ transports statistics associated to patterns of length three, and for each of them we provide generating functions for the distribution and popularity.

We need the following notations. For a step $X \in\{U, D, F\}$, an occurrence of the pattern $X^{+}$inside a path $P$ is an occurrence of $X^{k}$, where $X^{k}$ consists of $k$ consecutive repetitions of $X$, for some $k \geqslant 1$. The associated statistic of $X^{+}$(denoted $\left.\mathrm{X}^{+}\right)$will be equal to $\sum_{k \geqslant 1} \mathrm{X}^{k}$ where $\mathrm{X}^{k}$ is the statistic giving the number of occurrences of $X^{k}$. More generally, for two possibly empty sequences of steps $Y$ and $Z$ we define the pattern $Y X^{+} Z$ as patterns of the form $Y X^{k} Z$ for $k \geqslant 1$, and its associated statistic as $\mathrm{YX}^{+} \mathrm{Z}=\sum_{k \geqslant 1} \mathrm{YX}^{\mathrm{k}} \mathrm{Z}$. For instance, an occurrence of the pattern $D U^{+} D$ can be an occurrence of $D U D, D U U D$, $D U U U D$, and so on... In the path UUFDUUDFDUDUUUDDFFDD we count three occurrences of the pattern $D U^{+} D$. A path contains a dotted pattern $\bullet Y$ (resp. $Y \bullet$ ) if and only if it starts (resp. ends) with $Y$, and we use the notations $\bullet \mathrm{Y}, \mathrm{Y} \bullet$ for the associated statistics.

Any pattern $U U$ in a Dyck path is immediately followed by an up step or a down step, implying the statistic equation $\mathrm{UU}=\mathrm{UUU}+\mathrm{UUD}$. Also, any pattern $U U$ in a Dyck path $P$ is either at the beginning of $P$ or immediately preceded by an up or a down step, so UU $=\bullet U U+$ UUU + DUU. Using similar arguments we obtain the two following systems of statistic equations. 
$(a)\left\{\begin{array}{l}\mathrm{UU}=\mathrm{UUU}+\mathrm{UUD} \\ \mathrm{UU}=\mathrm{UUU}+\mathrm{DUU}+\bullet \mathrm{UU} \\ \mathrm{UD}=\mathrm{UUD}+\mathrm{DUD}+\bullet \mathrm{UD} \\ \mathrm{DU}=\mathrm{DUU}+\mathrm{DUD}\end{array}, \quad(b)\left\{\begin{array}{l}\mathrm{DD}=\mathrm{DDD}+\mathrm{UDD} \\ \mathrm{DD}=\mathrm{DDD}+\mathrm{DDU}+\mathrm{DD} \bullet \\ \mathrm{UD}=\mathrm{UDD}+\mathrm{UDU}+\mathrm{UD} \bullet \\ \mathrm{DU}=\mathrm{DDU}+\mathrm{UDU}\end{array}\right.\right.$.

Observe that for any $P \in \mathcal{D}_{n}^{h, \geqslant}, n \geqslant 1$, we have $\bullet \operatorname{UD}(P)=1$ (resp. -UU $(P)=0)$ when $P=(U D)^{n}$, and $\bullet \mathrm{UD}(P)=0($ resp. $\bullet \mathrm{UU}(P)=1)$ otherwise. So, we have the statistic equations $\phi(\bullet \mathrm{UD})=\delta_{F^{n}}$ and $\phi(\bullet \mathrm{UU})=$ $1-\phi(\bullet$ UD $)$, where $\delta_{F^{n}}$ is the Dirac statistic defined by $\delta_{F^{n}}(P)=1$ whenever $P=F^{n}$, and 0 otherwise.

Knowing the image through $\phi$ of only one statistic $\mathrm{X} \in\{\mathrm{UUU}$, UUD, DUU, DUD $\}$ and using results from Section 2, we can obtain the expressions of the images of the three other statistics from the system $(a)$. The same reasoning holds for the second system $(b)$. So, we split Section 3 into two subsections, each dealing with one of the two systems.

\subsection{The patterns $U U U, U U D, D U U, D U D$}

Theorem 3. For $n \geqslant 0$, the bijection $\phi$ from $\mathcal{D}_{n}^{h} \geqslant$ to $\mathcal{M}_{n}$ transports the statistic UUD as follows:

$$
\phi(\mathrm{UUD})=\mathrm{UF}^{+} \mathrm{D}+\mathrm{UD} .
$$

Proof. We proceed by induction on $n$. For $n=1$, we have $\operatorname{UUD}(U D)=0$. Since $\phi(U D)=F$, we obtain $\mathrm{UF}^{+} \mathrm{D}(F)+\mathrm{UD}(F)=0$, and the result holds. We assume the result for $k \leqslant n$, and we will prove it for $n+1$ by distinguishing two main cases.

- Whenever $P=\alpha U D$, we have $\phi(P)=\phi(\alpha) F$, and $\left(\mathrm{UF}^{+} \mathrm{D}+\mathrm{UD}\right)(\phi(P))=$ $\left(\mathrm{UF}^{+} \mathrm{D}+\mathrm{UD}\right)(\phi(\alpha))$. Using induction hypothesis, $\left(\mathrm{UF}^{+} \mathrm{D}+\mathrm{UD}\right)(\phi(\alpha))=$ $\operatorname{UUD}(\alpha)$ which is also equal to $\operatorname{UUD}(\alpha U D))$, proving the first case.

- Whenever $P=\alpha U U \beta D \gamma D$, we have $\phi(P)=\phi(\alpha) \phi(\gamma) U \phi(\beta) D$, and $\left(\mathrm{UF}^{+} \mathrm{D}+\mathrm{UD}\right)(\phi(P))=\left(\mathrm{UF}^{+} \mathrm{D}+\mathrm{UD}\right)(\phi(\alpha))+\left(\mathrm{UF}^{+} \mathrm{D}+\mathrm{UD}\right)(\phi(\gamma) U \phi(\beta) D) . \mathrm{Us}^{-}$ ing induction hypothesis, we obtain $\left(\mathrm{UF}^{+} \mathrm{D}+\mathrm{UD}\right)(\phi(P))=\mathrm{UUD}(\alpha)+\left(\mathrm{UF}^{+} \mathrm{D}+\right.$ $\mathrm{UD})(\phi(\gamma) U \phi(\beta) D)$. Now we distinguish three subcases in order to prove that $\left(\mathrm{UF}^{+} \mathrm{D}+\mathrm{UD}\right)(\phi(\gamma) U \phi(\beta) D)=\mathrm{UUD}(U U \beta D \gamma D)$.

If $\beta$ is the empty path, then $\left(\mathrm{UF}^{+} \mathrm{D}+\mathrm{UD}\right)(\phi(\gamma) U \phi(\beta) D)=1+\left(\mathrm{UF}^{+} \mathrm{D}+\right.$ $\mathrm{UD})(\phi(\gamma))=1+\operatorname{UUD}(\gamma)=\operatorname{UUD}(U U D \gamma D)$.

If $\beta=(U D)^{k}$ for some $k \geqslant 1$, then $\left(\mathrm{UF}^{+} \mathrm{D}+\mathrm{UD}\right)\left(\phi(\gamma) U \phi\left((U D)^{k}\right) D\right)=$ $\left(\mathrm{UF}^{+} \mathrm{D}+\mathrm{UD}\right)\left(\phi(\gamma) U F^{k} D\right)=\left(\mathrm{UF}^{+} \mathrm{D}+\mathrm{UD}\right)(\phi(\gamma))+1=\mathrm{UUD}(\gamma)+1=$ $\mathrm{UUD}\left(U U(U D)^{k} D \gamma D\right)=\mathrm{UUD}(U U \beta D \gamma D)$.

If $\beta$ starts with a double rise $U U$, then $\phi(\beta)$ contains at least one up step $\left(\phi(\beta) \neq F^{\ell}\right.$ for any $\left.\ell \geqslant 0\right)$. So, we have $\left(\mathrm{UF}^{+} \mathrm{D}+\mathrm{UD}\right)(\phi(\gamma) U \phi(\beta) D)=$ $\left(\mathrm{UF}^{+} \mathrm{D}+\mathrm{UD}\right)(\phi(\gamma))+\left(\mathrm{UF}^{+} \mathrm{D}+\mathrm{UD}\right)(\phi(\beta))=\mathrm{UUD}(\gamma)+\mathrm{UUD}(\beta)$, which equals to $\operatorname{UUD}(U U \beta D \gamma D)$.

Considering these three cases, the second case is proved and the induction is completed.

Theorem 4. For $n \geqslant 0$, the bijection $\phi$ from $\mathcal{D}_{n}^{h, \geqslant}$ to $\mathcal{M}_{n}$ transports the statistic UUU as follows:

$$
\phi(\mathrm{UUU})=\mathrm{UF}^{+} \mathrm{D}+2\left(\mathrm{UF}^{+} \mathrm{U}+\mathrm{UU}\right) .
$$


Proof. Using Theorem 3 and the equation UU $=$ UUD + UUU of system $(a)$, we have $\phi(\mathrm{UUU})=\phi(\mathrm{UU})-\phi(\mathrm{UUD})=\phi(\mathrm{UU})-\mathrm{UF}^{+} \mathrm{D}-\mathrm{UD}$, and using Theorem 1, we have $\phi(\mathrm{UUU})=\mathrm{U}+\mathrm{UU}+\mathrm{UF}-\mathrm{UF}^{+} \mathrm{D}-\mathrm{UD}$. Since we have $\mathrm{U}=\mathrm{UD}+\mathrm{UU}+\mathrm{UF}$ on $\mathcal{M}_{n}$, we obtain $\phi(\mathrm{UUU})=2(\mathrm{UU}+\mathrm{UF})-\mathrm{UF}^{+} \mathrm{D}$. Using the trivial equation $\mathrm{UF}=\mathrm{UF}^{+} \mathrm{D}+\mathrm{UF}^{+} \mathrm{U}$ for Motzkin paths, we complete the proof.

Theorem 5. For $n \geqslant 1$, the bijection $\phi$ from $\mathcal{D}_{n}^{h, \geqslant}$ to $\mathcal{M}_{n}$ transports the statistic DUU as follows:

$$
\phi(\mathrm{DUU})=\mathrm{UF}^{+} \mathrm{D}+\mathrm{UD}+\delta_{F^{n}}-1,
$$

where $\delta_{F^{n}}$ is the Dirac statistic defined by $\delta_{F^{n}}(P)=1$ whenever $P=F^{n}$, and 0 otherwise.

Proof. Using the difference of the first two equations of $(a)$, we obtain DUU $=$ $\mathrm{UUD}-\bullet \mathrm{UU}$, and finally, $\phi(\mathrm{DUU})=\phi(\mathrm{UUD})-\phi(\bullet \mathrm{UU})=\mathrm{UF}^{+} \mathrm{D}+\mathrm{UD}-\phi(\bullet \mathrm{UU})$. As discussed at the beginning of Section $3, \phi(\bullet U U)=1-\delta_{F^{n}}$, which completes the proof.

Theorem 6. For $n \geqslant 1$, the bijection $\phi$ from $\mathcal{D}_{n}^{h, \geqslant}$ to $\mathcal{M}_{n}$ transports the statistic DUD as follows:

$$
\phi(\mathrm{DUD})=\mathrm{F}-\mathrm{UF}^{+} \mathrm{D}-\delta_{F^{n}}
$$

Proof. We have DUD $=$ UD - UUD $-\bullet U D$, which implies that $\phi($ DUD $)=$ $\phi(\mathrm{UD})-\phi(\mathrm{UUD})-\phi(\bullet \mathrm{UD})$. From Theorem 1 , Theorem 3 and the observations after the systems $(a)$ and $(b)$ we obtain $\phi(\mathrm{DUD})=\mathrm{UD}+\mathrm{F}-\mathrm{UD}-\mathrm{UF}^{+} \mathrm{D}-\delta_{F^{n}}=$ $\mathrm{F}-\mathrm{UF}^{+} \mathrm{D}-\delta_{F^{n}}$.

Theorem 7. The bivariate generating functions $F_{p}(x, y)$ where the coefficient of $x^{n} y^{k}$ is the number of Dyck paths in $\mathcal{D}_{n}^{h, \geqslant}$ containing exactly $k$ occurrences of the pattern $p \in\{U U U, U U D, D U U, D U D\}$ are given by the following expressions listed in the same order as written above.

$$
\begin{gathered}
\begin{array}{c}
\frac{x^{3} y-x^{3}-x^{2} y^{2}+2 x-1+\sqrt{(x-x y-1)\left(x^{2}-x y+x-1\right)\left(x^{3}-x^{3} y+x^{2} y^{2}+2 x^{2} y-2 x y-2 x+1\right)}}{2 x^{2} y^{2}(x-1)}, \\
\frac{x^{2} y-2 x^{2}+2 x-1+\sqrt{\left(x^{2} y-1\right)\left(x^{2} y-4 x^{2}+4 x-1\right)}}{2 x^{2}(x-1)}, \\
\frac{2 x-x^{2} y-1+\sqrt{\left(x^{2} y-1\right)\left(x^{2} y-4 x^{2}+4 x-1\right)}}{2 x^{2} y(x-1)}, \\
\frac{x^{3} y-x^{3}-x^{2} y^{2}+2 x y-1+\sqrt{(x y-x-1)\left(x^{2}+y x-x-1\right)\left(x^{3} y-x^{3}+x^{2} y^{2}+2 x^{2} y-2 x y-2 x+1\right)}}{2 x^{2}(x y-1)} .
\end{array} .
\end{gathered}
$$

Proof. For $p=U U U$ and using Theorem 4, we have $\phi(\mathrm{UUU})=\mathrm{UF}^{+} \mathrm{D}+$ $2\left(\mathrm{UF}^{+} \mathrm{U}+\mathrm{UU}\right)$. So, we decompose Motzkin paths according to the patterns $U F^{+} D, U F^{+} U$, and $U U$ in order to exhibit a functional equation having $F_{p}(x, y)$ as solution:

$$
\mathcal{M}=\epsilon+F \mathcal{M}+U D \mathcal{M}+U \mathcal{M}_{0} D \mathcal{M}+U\left(\mathcal{M} \backslash\left(\mathcal{M}_{0} \cup \epsilon\right)\right) D \mathcal{M}
$$


where $\mathcal{M}_{0}$ is the subset of $\mathcal{M}$ consisting of paths of the form $F^{k}$ for $k \geqslant 1$. The generating function for $\epsilon+F \mathcal{M}+U D \mathcal{M}$ is $1+x F_{U U U}(x, y)+x^{2} F_{U U U}(x, y)$; the g.f. for $U \mathcal{M}_{0} D \mathcal{M}$ is $x^{2} y \frac{x}{1-x} F_{U U U}(x, y)$ since a path in $U \mathcal{M}_{0} D$ is an occurrence of $U F^{+} D$; the g.f. for $U\left(\mathcal{M} \backslash\left(\mathcal{M}_{0} \cup \epsilon\right)\right) D \mathcal{M}$ is $x^{2} y^{2}\left(F_{U U U}(x, y)-\frac{1}{1-x}\right) F_{U U U}(x, y)$ since a path in $U\left(\mathcal{M} \backslash\left(\mathcal{M}_{0} \cup \epsilon\right)\right) D$ starts with either an occurrence of $U U$ or an occurrence of $U F^{+} U$.

So the functional equation is:

$$
\begin{aligned}
F_{U U U}(x, y)=1+x F_{U U U}(x, y) & +x^{2} F_{U U U}(x, y)+x^{2} y \frac{x}{1-x} F_{U U U}(x, y)+ \\
& +x^{2} y^{2}\left(F_{U U U}(x, y)-\frac{1}{1-x}\right) F_{U U U}(x, y) .
\end{aligned}
$$

A simple calculation (with Maple for instance) provides the result.

All other generating functions are obtained using a similar method, so we do not give the proofs here.

The generating function $G_{p}(x)$ for the popularity of the pattern $p \in\{U U U, U U D$, $D U U, D U D\}$ is obtained directly by evaluating $\left.\frac{\partial F_{p}(x, y)}{\partial y}\right|_{y=1}$. Table 3 provides the first terms of the associated sequences. See also Table 4 for an illustration of the distribution of $p \in\{U U U, U U D, D U U, D U D\}$.

\begin{tabular}{c|c|c} 
Pattern & Popularity sequence & OEIS \\
\hline UUU & $0,0,1,5,19,65,210,658,2023,6147,18534,55594$ & \\
UUD & $0,1,3,9,25,70,196,553,1569,4476,12826,36894$ & A097861 \\
DUU & $0,0,0,1,5,20,70,231,735,2289,7029,21384$ & A304011 ? \\
DUD & $0,1,4,12,35,100,286,819,2353,6780,19591,56749$ &
\end{tabular}

Table 3: Popularity of $p \in\{U U U, U U D, D U U, D U D\}$ in $\mathcal{D}_{n}^{h, \geqslant}$ for $1 \leqslant n \leqslant 12$.

The popularity sequence for $U U D$ in $\mathcal{D}_{n}^{h, \geqslant}$ is the sequence A097861, corresponding to the number of humps in all Motzkin paths of length $n$ (a hump equals $U F^{+} D$ or $U D$ in our notation). The popularity for $D U U$ seems to generate the sequence A304011, but we did not succeed in proving this fact, so we leave it as a conjecture. 


\begin{tabular}{c|ccccccccc}
$k \backslash n$ & 1 & 2 & 3 & 4 & 5 & 6 & 7 & 8 & 9 \\
\hline 0 & 1 & 2 & 3 & 5 & 8 & 13 & 21 & 34 & 55 \\
1 & & & 1 & 3 & 8 & 18 & 38 & 76 & 147 \\
2 & & & & 1 & 4 & 14 & 40 & 104 & 250 \\
3 & & & & & 1 & 5 & 21 & 71 & 215 \\
4 & & & & & & 1 & 6 & 30 & 119 \\
5 & & & & & & & 1 & 7 & 40
\end{tabular}

(a) $U U U$

\begin{tabular}{c|ccccccccc}
$k \backslash n$ & 1 & 2 & 3 & 4 & 5 & 6 & 7 & 8 & 9 \\
\hline 0 & 1 & 1 & 1 & 1 & 1 & 1 & 1 & 1 & 1 \\
1 & & 1 & 3 & 7 & 15 & 31 & 63 & 127 & 255 \\
2 & & & & 1 & 5 & 18 & 56 & 160 & 432 \\
3 & & & & & & 1 & 7 & 34 & 138 \\
4 & & & & & & & & 1 & 9
\end{tabular}

(c) $U U D$

\begin{tabular}{c|ccccccccc}
$k \backslash n$ & 1 & 2 & 3 & 4 & 5 & 6 & 7 & 8 & 9 \\
\hline 0 & 1 & 1 & 1 & 2 & 3 & 6 & 10 & 20 & 36 \\
1 & & 1 & 2 & 3 & 7 & 13 & 30 & 58 & 130 \\
2 & & & 1 & 3 & 6 & 16 & 35 & 91 & 199 \\
3 & & & & 1 & 4 & 10 & 30 & 75 & 216 \\
4 & & & & & 1 & 5 & 15 & 50 & 140 \\
5 & & & & & & 1 & 6 & 21 & 77
\end{tabular}

(b) $D U D$

\begin{tabular}{c|ccccccccc}
$k \backslash n$ & 1 & 2 & 3 & 4 & 5 & 6 & 7 & 8 & 9 \\
\hline 0 & 1 & 2 & 4 & 8 & 16 & 32 & 64 & 128 & 256 \\
1 & & & & 1 & 5 & 18 & 56 & 160 & 432 \\
2 & & & & & & 1 & 7 & 34 & 138 \\
3 & & & & & & & & 1 & 9 \\
4 & & & & & & & & &
\end{tabular}

(d) $D U U$

Table 4: Number of paths from $\mathcal{D}_{n}^{h, \geqslant}$ having $k$ occurrences of the considered pattern.

Dyck paths from $\mathcal{D}_{n}^{h, \geqslant}$ avoiding $U U U, D U U$ respectively generate Fibonacci numbers and integer squares. Those avoiding $D U D$ seem to correspond to A007562 (number of planted trees where non-root, non-leaf nodes at even distance from root are of degree 2). At the present time, there is no closed form for the generating function of sequence A007562. Note that any path avoiding $D U U$ has at most one occurrence of $U U D$. Also, Dyck paths containing two occurrences of $U U D$ in $\mathcal{D}_{n}^{h, \geqslant}$ generate a shift of sequence A001793, which corresponds to a subsequence in the triangle of coefficients of Chebyshev's polynomials which is sequence A053120.

\subsection{The patterns $D D D, D D U, U D D, U D U$}

Theorem 8. For $n \geqslant 0$, the bijection $\phi$ from $\mathcal{D}_{n}^{h} \geqslant$ to $\mathcal{M}_{n}$ transports the statistic UDU as follows:

$$
\phi(\mathrm{UDU})=\mathrm{FF}+\mathrm{FUD}
$$

Proof. We proceed by induction on $n$. For $n=1$, we have $\operatorname{UDU}(U D)=0$. With $\phi(U D)=F$, we obtain $\mathrm{FF}(F)+\operatorname{FUD}(F)=0$, and the result holds. We assume the result for $k \leqslant n$, and we will prove it for $n+1$.

- Whenever $P=\alpha U D$, we have $\phi(P)=\phi(\alpha) F$, and (FF + FUD) $(\phi(P))=$ $\mathrm{FF}(\phi(\alpha) F)+\mathrm{FUD}(\phi(\alpha) F)=\mathrm{FF}(\phi(\alpha) F)+\mathrm{FUD}(\phi(\alpha))$. We distinguish two cases: $(i) \phi(\alpha)$ ends with $F$, and $(i i)$ otherwise. In the case $(i), \alpha$ ends with $U D$, and thus (FF + FUD $)(\phi(P))=1+\mathrm{FF}(\phi(\alpha))+\mathrm{FUD}(\phi(\alpha))$. Using the induction hypothesis we have $(\mathrm{FF}+\mathrm{FUD})(\phi(P))=1+\mathrm{UDU}(\alpha)=\mathrm{UDU}(\alpha U D)=$ $\mathrm{UDU}(P)$. In the case $(i i), \alpha$ does not end with $U D$, and thus $\phi(\alpha)$ does not end 
with $F$. So, we have $(\mathrm{FF}+\mathrm{FUD})(\phi(P))=\mathrm{FF}(\phi(\alpha))+\mathrm{FUD}(\phi(\alpha))$, and using the induction hypothesis $(\mathrm{FF}+\mathrm{FUD})(\phi(P))=\mathrm{UDU}(\alpha)=\mathrm{UDU}(\alpha U D)=\mathrm{UDU}(P)$.

- Whenever $P$ does not end with $U D$, we have $P=\alpha U U \beta D \gamma D$, and $(\mathrm{FF}+\mathrm{FUD})(\phi(P))=(\mathrm{FF}+\mathrm{FUD})(\phi(\alpha) \phi(\gamma) U \phi(\beta) D)$. Note that $\alpha$ cannot end with $U D$, otherwise it would contradict $P \in \mathcal{D}_{n}^{h, \geqslant}$. This means that $\phi(\alpha)$ cannot end with $F$. So, all the possible occurrences of $F F$ in $\phi(P)$ belong necessarily to $\phi(\alpha), \phi(\beta)$ and $\phi(\gamma)$, which implies that $\operatorname{FF}(\phi(P))=$ $\mathrm{FF}(\phi(\alpha))+\mathrm{FF}(\phi(\beta))+\mathrm{FF}(\phi(\gamma))$. On the other hand, the possible occurrences of FUD in $\phi(P)$ belong necessarily to $\phi(\alpha), \phi(\beta), \phi(\gamma)$, and eventually at the junction of $\phi(\gamma)$ and $U \phi(\beta) D$ whenever $\phi(\gamma)$ ends with $F$ and $\phi(\beta)=\epsilon$. So, we distinguish two cases: $(a) \phi(\gamma)$ ends with $F$ and $\beta=\epsilon$, and $(b)$ otherwise. In the case $(a)$, we have $\operatorname{FUD}(\phi(P))=1+\mathrm{FF}(\phi(\alpha))+\mathrm{FF}(\phi(\beta))+\mathrm{FF}(\phi(\gamma))+$ $\operatorname{FUD}(\phi(\alpha))+\operatorname{FUD}(\phi(\beta))+\operatorname{FUD}(\phi(\gamma))$, and using the induction hypothesis, we obtain $\operatorname{FUD}(\phi(P))=1+\mathrm{UDU}(\alpha)+\mathrm{UDU}(\beta)+\mathrm{UDU}(\gamma)=\mathrm{UDU}(\alpha U U \beta D \gamma D)=$ $\mathrm{UDU}(P)$. In the case $(b)$, we have $\operatorname{FUD}(\phi(P))=\mathrm{FF}(\phi(\alpha))+\mathrm{FF}(\phi(\beta))+$ $\mathrm{FF}(\phi(\gamma))+\mathrm{FUD}(\phi(\alpha))+\mathrm{FUD}(\phi(\beta))+\mathrm{FUD}(\phi(\gamma))$, and using the induction hypothesis, we obtain $\operatorname{FUD}(\phi(P))=\mathrm{UDU}(\alpha)+\mathrm{UDU}(\beta)+\mathrm{UDU}(\gamma)=\mathrm{UDU}(\alpha U U \beta D \gamma D)=$ $\mathrm{UDU}(P)$. So, the proof is complete.

Theorem 9. For $n \geqslant 0$, the bijection $\phi$ from $\mathcal{D}_{n}^{h, \geqslant}$ to $\mathcal{M}_{n}$ transports the statistic UDD as follows:

$$
\phi(\mathrm{UDD})=\mathrm{FD}+\mathrm{UD}+\mathrm{FUU}+\mathrm{FUF} .
$$

Proof. Considering the third equation of system $(b)$, we have $\phi(\mathrm{UDD})=\phi(\mathrm{UD})-$ $\phi(\mathrm{UDU})-\phi(\mathrm{UD \bullet})$. Using Theorems 1 and 8 , we obtain $\phi(\mathrm{UDD})=\mathrm{F}+$ $\mathrm{UD}-\mathrm{FF}-\mathrm{FUD}-\phi(\mathrm{UD} \bullet)$. In any Motzkin path $P$, a flat step is either at the end of $P$, or followed by $F$, or $D$, or $U U$, or $U D$, or $U F$, that is $\mathrm{F}=\mathrm{F} \bullet+\mathrm{FF}+\mathrm{FD}+\mathrm{FUU}+\mathrm{FUD}+\mathrm{FUF}$. Then, we obtain $\phi(\mathrm{UDD})=$ $\mathrm{F} \bullet+\mathrm{UD}+\mathrm{FD}+\mathrm{FUU}+\mathrm{FUF}-\phi(\mathrm{UD} \bullet)$. Using the definition of $\phi$ in Introduction, it is clear that $\phi$ transports $\mathrm{UD} \bullet$ into $\mathrm{F} \bullet$, and the result holds.

Theorem 10. For $n \geqslant 0$, the bijection $\phi$ from $\mathcal{D}_{n}^{h, \geqslant}$ to $\mathcal{M}_{n}$ transports the statistic DDU as follows:

$$
\phi(\mathrm{DDU})=\mathrm{DF}+\mathrm{DU}+\mathrm{FUU}+\mathrm{FUF} .
$$

Proof. Combining the fourth equation DU $=$ DDU + UDU of system $(b)$ with Theorems 1 and 8, we obtain $\phi(\mathrm{DDU})=\mathrm{FF}+\mathrm{FU}+\mathrm{DF}+\mathrm{DU}-\mathrm{FF}-\mathrm{FUD}=$ $\mathrm{FU}+\mathrm{DF}+\mathrm{DU}-\mathrm{FUD}$. The proof is completed using the straightforward equation $\mathrm{FU}=\mathrm{FUU}+\mathrm{FUF}+\mathrm{FUD}$ on Motzkin paths.

Theorem 11. For $n \geqslant 0$, the bijection $\phi$ from $\mathcal{D}_{n}^{h, \geqslant}$ to $\mathcal{M}_{n}$ transports the statistic DDD as follows:

$$
\phi(\mathrm{DDD})=2(\mathrm{UU}+\mathrm{UF})-\mathrm{FD}-\mathrm{FUU}-\mathrm{FUF} .
$$


Proof. Combining the first equation DD $=$ DDD + UDD of system $(b)$ with Theorems 1 and 9, we obtain $\phi(D D D)=U+U U+U F-F D-U D-F U U-F U F$. Using $\mathrm{U}=\mathrm{UF}+\mathrm{UD}+\mathrm{UU}$ on Motzkin paths, the result holds.

Theorem 12. The bivariate generating functions $F_{p}(x, y)$ where the coefficient of $x^{n} y^{k}$ is the number of Dyck paths in $\mathcal{D}_{n}^{h, \geqslant}$ containing exactly $k$ occurrences of the pattern $p \in\{U D U, U D D, D D U, D D D\}$ are given by the following expressions listed in the same order as written above.

$$
\begin{gathered}
\frac{1+x\left(x^{2}-x^{2} y-y\right)-\sqrt{A}}{2 x^{2}(x-x y+1)}, \\
\frac{1+x\left(x^{2} y-x^{2}-x y+x-1\right)-\sqrt{B}}{2 x^{2}(x y-x+1)^{2}} \\
\frac{1+x\left(2 x^{2} y^{2}-3 x^{2} y+x^{2}+x y-x-1\right)-\sqrt{C}}{2 x^{2} y(x y-x+1)} \\
\frac{1-x\left(x^{2} y^{2}-x^{2} y-x y^{2}+x+1\right)-\sqrt{D}}{2 x^{2}(x y-x-y)^{2}}
\end{gathered}
$$

where

$$
\begin{gathered}
A=(x+1)\left(x^{2} y-x^{2}+x y-x-1\right)\left(x^{3} y-x^{3}-2 x^{2} y+2 x^{2}+x y+2 x-1\right), \\
B=(x+1)\left(x^{2} y-x^{2}+1\right)\left(x^{3} y-x^{3}-3 x^{2} y+3 x^{2}-3 x+1\right), \\
C=(x+1)\left(x^{2} y-x^{2}+1\right)\left(x^{3} y-x^{3}-3 x^{2} y+3 x^{2}-3 x+1\right), \\
D=(x y+1)\left(x^{2} y-x^{2}-x y+x-1\right)\left(x^{3} y^{2}-x^{3} y-x^{2} y^{2}-2 x^{2} y+3 x^{2}+2 x y+x-1\right) .
\end{gathered}
$$

Proof. Let $A_{p}(x, y)$ (resp. $B_{p}(x, y)$ ) be the bivariate generating function where the coefficient of $x^{n} y^{k}$ is the number of Motzkin paths of length $n$ ending with a flat step (resp. down step) having exactly $k$ occurrences of the patterns related to the statistic $\phi(p)$ obtained in the r.h.s. of equations of Theorems 8,9,10,11. Using a refinement of the classical decompositions of non-empty Motzkin paths by taking into account the occurrences of the considered patterns, we deduce functional equations for $A_{p}(x, y)$ and $B_{p}(x, y)$. The method being classic, we do not give any more details. The solutions are obtained by a simple calculation.

For $p=U D U$ :

$$
\begin{aligned}
& \left\{\begin{array}{l}
F_{p}(x, y)=1+A_{p}(x, y)+B_{p}(x, y) \\
A_{p}(x, y)=x+x y A_{p}(x, y)+x B_{p}(x, y) \\
B_{p}(x, y)=x^{2}+x^{2} y A_{p}(x, y)+x^{2} B_{p}(x, y)+x^{2} F_{p}(x, y)\left(F_{p}(x, y)-1\right) ;
\end{array}\right. \\
& \quad \begin{array}{l}
F_{p}(x, y)=1+A_{p}(x, y)+B_{p}(x, y) \\
A_{p}(x, y)=x F_{p}(x, y) \\
B_{p}(x, y)=x^{2} y F_{p}(x, y)+x^{2} y A_{p}(x, y)+x^{2} B_{p}(x, y)+ \\
+x^{2} B_{p}(x, y)^{2}+x^{2} y A_{p}(x, y) B_{p}(x, y)+x^{2} y^{2} A_{p}(x, y)^{2}+x^{2} y A_{p}(x, y) B_{p}(x, y) ;
\end{array}
\end{aligned}
$$

For $p=D D U$ : 


$$
\begin{aligned}
& \left\{\begin{array}{l}
F_{p}(x, y)=1+A_{p}(x, y)+B_{p}(x, y) \\
A_{p}(x, y)=x+x A_{p}(x, y)+x y B_{p}(x, y) \\
B_{p}(x, y)=x^{2} F_{p}(x, y)+x^{2} y A_{p}(x, y)\left(F_{p}(x, y)-1\right)+x^{2} A_{p}(x, y)+ \\
\quad+x^{2} y B_{p}(x, y) F_{p}(x, y)
\end{array}\right. \\
& \begin{array}{l}
\text { For } p=D D D: \\
F_{p}(x, y)=1+A_{p}(x, y)+B_{p}(x, y) \\
A_{p}(x, y)=x F_{p}(x, y) \\
B_{p}(x, y)=x^{2} F_{p}(x, y)+x^{2} y^{2} B_{p}(x, y)+x^{2} y^{2} A_{p}(x, y) / y+x^{2} y^{2} A_{p}(x, y)^{2} / y^{2}+ \\
+x^{2} y^{2} A_{p}(x, y) B_{p}(x, y) / y+x^{2} y^{2} B_{p}(x, y)^{2}+x^{2} y^{2} A_{p}(x, y) B_{p}(x, y) / y .
\end{array}
\end{aligned}
$$

The above equations are intentionally left in non-simplified forms, in order to allow the reader to easily retrieve the refined decompositions from the classical decomposition of Motzkin paths $\mathcal{M}=\epsilon+\mathcal{M} F+\mathcal{M} U \mathcal{M} D$.

Generating function $G_{p}(x)$ for the popularity of a pattern $p$ is obtained directly by evaluating $\left.\frac{\partial F_{p}(x, y)}{\partial y}\right|_{y=1}$. Table 6 provides the first terms of the generated sequences. See also Table 5 for an illustration of the distribution of $p \in\{U D U, U D D, D D U, D D D\}$.

Unlike what happens for classical Dyck paths, the popularity of $U D U$ in $\mathcal{D}_{n+1}^{h, \geqslant}$ is equal to the popularity of $U D$ in $\mathcal{D}_{n}^{h, \geqslant}$, while the corresponding dis-

\begin{tabular}{|c|c|c|c|c|c|c|c|c|c|c|c|c|c|c|c|c|c|c|}
\hline$k \backslash n$ & 12 & 3 & 4 & 5 & 6 & 7 & 8 & 9 & $k \backslash n$ & 1 & 2 & 3 & 4 & 5 & 6 & 7 & 8 & 9 \\
\hline 0 & 12 & 3 & 6 & 11 & 22 & 43 & 87 & 176 & 0 & 1 & 1 & 2 & 4 & 8 & 17 & 37 & 82 & 185 \\
\hline 1 & & 1 & 2 & 7 & 16 & 43 & 102 & 251 & 1 & & 1 & 1 & 3 & 7 & 17 & 41 & 102 & 252 \\
\hline 2 & & & 1 & 2 & 10 & 25 & 80 & 208 & 2 & & & 1 & 1 & 4 & 10 & 28 & 73 & 200 \\
\hline 3 & & & & 1 & 2 & 13 & 34 & 130 & 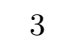 & & & & 1 & 1 & 5 & 13 & 41 & 113 \\
\hline 4 & & & & & 1 & 2 & 17 & 46 & 4 & & & & & & 1 & 6 & 16 & 56 \\
\hline 5 & & & & & & 1 & 2 & 21 & 5 & & & & & & 1 & 1 & 7 & 19 \\
\hline
\end{tabular}
tributions are different. Dyck paths from $\mathcal{D}_{n}^{h, \geqslant}$ avoiding a pattern $U D U$ (resp. $D D D$ ) are counted by the Generalized Catalan numbers (resp. by the numbers of ordered trees with $n$ edges and having no branches of length 1), which corresponds to the sequence A004148 (resp. A026418).

(a) $D D D$

(b) $U D U$

\begin{tabular}{c|ccccccccc}
$k \backslash n$ & 1 & 2 & 3 & 4 & 5 & 6 & 7 & 8 & 9 \\
\hline 0 & 1 & 2 & 3 & 4 & 5 & 6 & 7 & 8 & 9 \\
1 & & & 1 & 5 & 14 & 31 & 59 & 102 & 164 \\
2 & & & & & 2 & 14 & 57 & 174 & 444 \\
3 & & & & & & & 4 & 39 & 209 \\
4 & & & & & & & & & 9
\end{tabular}

(c) $D D U$

\begin{tabular}{c|ccccccccc}
$k \backslash n$ & 1 & 2 & 3 & 4 & 5 & 6 & 7 & 8 & 9 \\
\hline 0 & 1 & 1 & 1 & 1 & 1 & 1 & 1 & 1 & 1 \\
1 & & 1 & 3 & 6 & 10 & 15 & 21 & 28 & 36 \\
2 & & & & 2 & 10 & 31 & 75 & 156 & 292 \\
3 & & & & & 4 & 30 & 129 & 417 \\
4 & & & & & & & 9 & 89
\end{tabular}

(d) $U D D$

Table 5: Number of paths from $\mathcal{D}_{n}^{h, \geqslant}$ having $k$ occurences of the considered pattern. 


\begin{tabular}{c|c|c} 
Pattern & Popularity sequence & OEIS \\
\hline DDD & $0,0,1,4,14,46,145,448,1365,4124,12387,37060$ & \\
DDU & $0,0,1,5,18,59,185,567,1715,5146,15363,45715$ & \\
UDD & $0,1,3,10,30,89,261,763,2227,6499,18973,55428$ & \\
UDU & $0,1,3,8,22,61,171,483,1373,3923,11257,32418$ & A025566
\end{tabular}

Table 6: Popularity of 3 -length patterns in $\mathcal{D}_{n}^{h, \geqslant}$ for $1 \leqslant n \leqslant 12$.

\section{Acknowledgements}

We would like to thank the anonymous referees for their helpful comments and suggestions.

\section{References}

[1] A. Bacher, A. Bernini, L. Ferrari, B. Gundy, R. Pinzani, and J. West. The Dyck pattern poset. Discrete Mathematics, 321:12-23, 2014.

[2] E. Barcucci, A. Del Lungo, S. Fezzi, and R. Pinzani. Nondecreasing Dyck paths and $q$-Fibonacci numbers. Discrete Mathematics, 170(1-3):211-217, 1997.

[3] E. Barcucci, R. Pinzani, R. Sprugnoli, et al. The Motzkin family. Pure Mathematics and Applications, 2(3-4):249-279, 1992.

[4] J.-L. Baril, S. Kirgizov, and A. Petrossian. Dyck paths with a first return decomposition constrained by height. Discrete Mathematics, 341(6):1620 $1628,2018$.

[5] J.-L. Baril, S. Kirgizov, and A. Petrossian. Motzkin paths with a restricted first return decomposition. Integers, 19, A46, 2019.

[6] C. Brennan and S. Mavhungu. Peaks and valleys in Motzkin paths. Quaestiones Mathematicae, 33(2):171-188, 2010.

[7] D. Callan. Two bijections for Dyck path parameters. arXiv preprint math/0406381, 2004.

[8] E. Czabarka, R. Flórez, and L. Junes. Some enumerations on nondecreasing Dyck paths. The Electronic Journal of Combinatorics, 22(1):13,2015 .

[9] A. Denise and R. Simion. Two combinatorial statistics on Dyck paths. Discrete Mathematics, 137(1-3):155-176, 1995.

[10] E. Deutsch. Dyck path enumeration. Discrete Mathematics, 204(1-3):167$202,1999$.

[11] R. Donaghey and L.W. Shapiro. Motzkin numbers. Journal of Combinatorial Theory, Series A, 23(3):291-301, 1977. 
[12] D. Drake and R. Gantner. Generating functions for plateaus in Motzkin paths. arXiv preprint arXiv:1109.3273, 2011.

[13] L. Ferrari and E. Munarini. Enumeration of edges in some lattices of paths. Journal of Integer Sequences, 17(2):3, 2014.

[14] S.M. Losanitsch. Die isomerie-arten bei den homologen der paraffin-reihe. Berichte der deutschen chemischen Gesellschaft, 30(2):1917-1926, 1897.

[15] T. Mansour. Counting peaks at height $k$ in a Dyck path. J. Integer Seq, $5(02.1): 1,2002$

[16] T. Mansour. Statistics on Dyck paths. J. Integer Seq, 9(1):06-1, 2006.

[17] T. Mansour, M. Schork, and Y. Sun. Motzkin numbers of higher rank: generating function and explicit expression. Journal of Integer Sequences, 10(2):3, 2007.

[18] D. Merlini, R. Sprugnoli, and M.C. Verri. Some statistics on Dyck paths. Journal of statistical planning and inference, 101(1-2):211-227, 2002.

[19] A. Panayotopoulos and A. Sapounakis. On the prime decomposition of Dyck words. Journal of Combinatorial Mathematics and Combinatorial Computing, 40:33-40, 2002.

[20] P. Peart and W.J. Woan. Dyck paths with no peaks at height $k$. J. Integer Seq, 4(1), 2001.

[21] A. Sapounakis, I. Tasoulas, and P. Tsikouras. Counting strings in Dyck paths. Discrete Mathematics, 307(23):2909-2924, 2007.

[22] A. Sapounakis and P. Tsikouras. Counting peaks and valleys in $k$-colored Motzkin paths. the electronic journal of combinatorics, 12(1):16, 2005.

[23] N.J.A. Sloane. The on-line encyclopedia of integer sequences. https://oeis.org, 2019.

[24] Y. Sun. The statistic "number of udu's" in Dyck paths. Discrete mathematics, 287(1-3):177-186, 2004.

[25] S. Wagner and H. Prodinger. Minimal and maximal plateau lengths in Motzkin paths. Discrete Mathematics \&3 Theoretical Computer Science, 2007. 